\title{
Stereophotomacrography to Illustrate Tire Failure Morphology
}

Ronald W. Smith

Consultant, 2450 Clarke Drive, Lake Havasu City, AZ 86403

The failure analyst gains first hand knowledge of failure modes; however, that knowledge will be of limited value until the presentation is given or the report is issued. The inclusion of stereoscopic images enhances the teaching value of presentations and reports. This is particularly important in the case of passenger tires that are composite products that often exhibit very complex three dimensional failure morphologies. This report shows how anaglyphs, generated using commercial software [1] coupled with Microsoft PowerPoint enhances the teaching value of reports.

Stereophotomacrographs are produced using a $35 \mathrm{~mm}$ film camera mounted on a rotating arm with the object located at the pivot position. A wide variety of stereo angles are available from $0^{\circ}$ thru $+/-15^{\circ}$, thus providing for hyper stereo effects that are sometimes required to enhance visual impacts. A bellows attachment with extension rings provides for macro views in the range of $10 \mathrm{X}$ on the final $4 \times 6$ anaglyph.

I prefer to mount the stereo pairs on standard $3 \times 7$ inch stereo cards suitable for viewing with a Holmes or similar viewer. It is quicker and easier to make anaglyphs by digitally scanning mounted stereo cards that have demonstrated the desired stereo effects. I routinely produce red-blue anaglyphs (red over right eye) from stereo pairs photographed during the examination of tire failures.

The morphologies most generally encountered in tire failures have been documented by the author [2]. Many failures appear to be "basket cases" from which no data could be extracted. Contrarily, there are failure patterns that are repeatable and can be categorized, i.e., belt edge separations, belt-leaving-belt catastrophes, effects due to severe aging, tie abuse, and road hazards. Examples of these morphologies are reproduced here as redblue anaglyphs but the color effects are not reproduced in the printed paper but are observable from the $\mathrm{CD}$ copy of the proceedings, platform presentation, and/or poster presentation.

The source of tire samples is the highways and byways within the tri-state corner of Arizona-California-Nevada. Whole tires, catastrophically failed tires and fragments from failed tires yield are collected and examined in the as-is state. Subsequent dissection yields insight about internal changes that occur within the tire prior to and leading up to failure. These objects are extremely three-dimensional and stereophotomacrography is relied upon routinely.

\section{References}

[1] 3D Stereo Image Factory TM PLUS by SOFTreat

[2] R.W. Smith, Rubber Chemistry \& Technology, 70, (1997) 283 


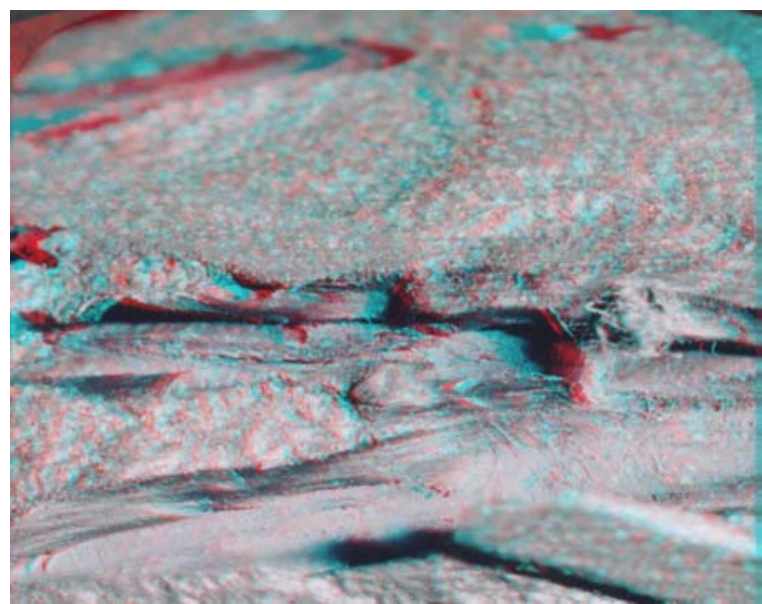

Fig 1. Tread Groove Cracking

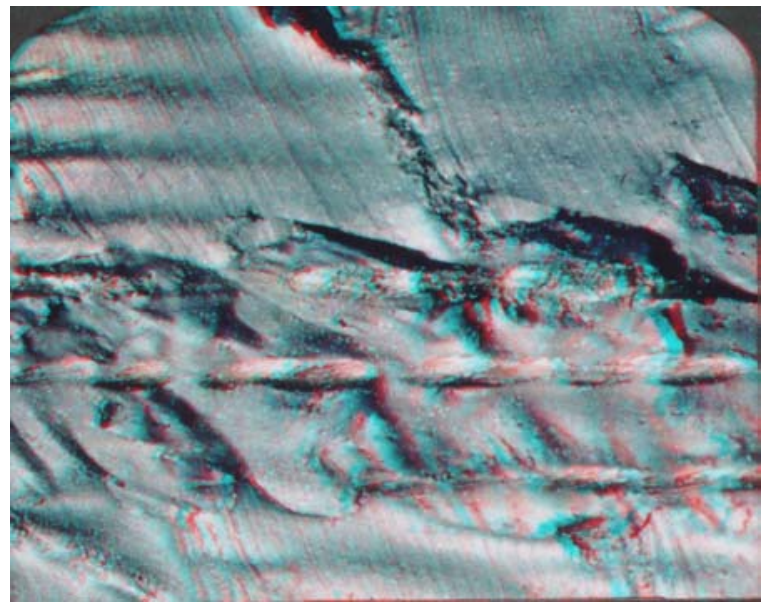

Fig 3. Juxtaposition: Organic-Steel Cords

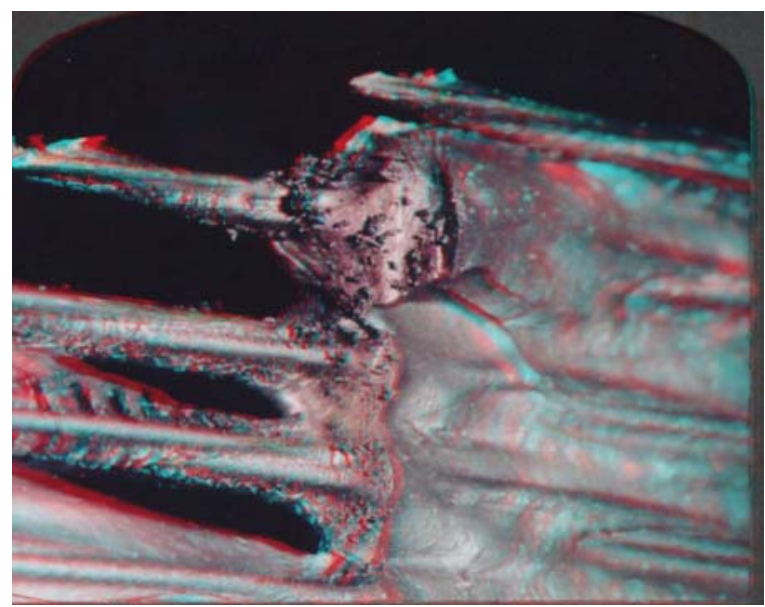

Fig 2. Wire Ends - Belt Edge Separation

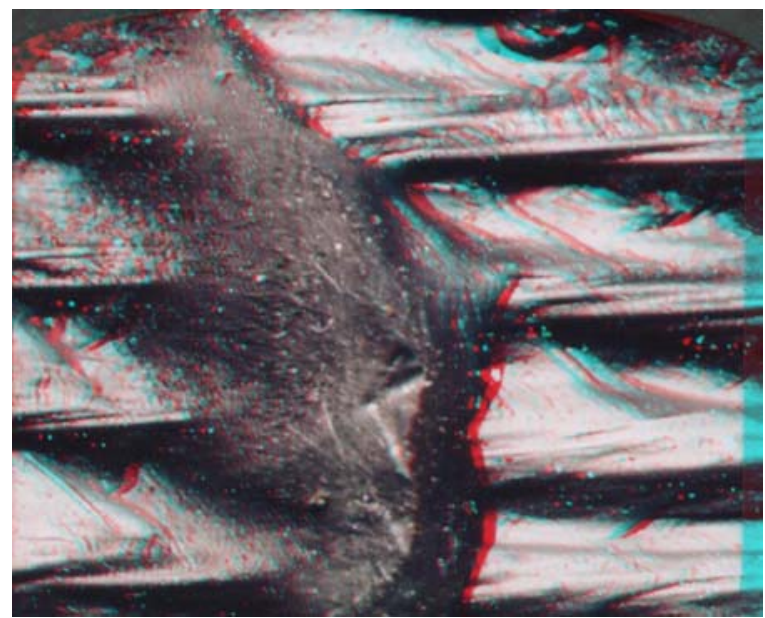

Fig 4 Between-Belt Tearing Lip

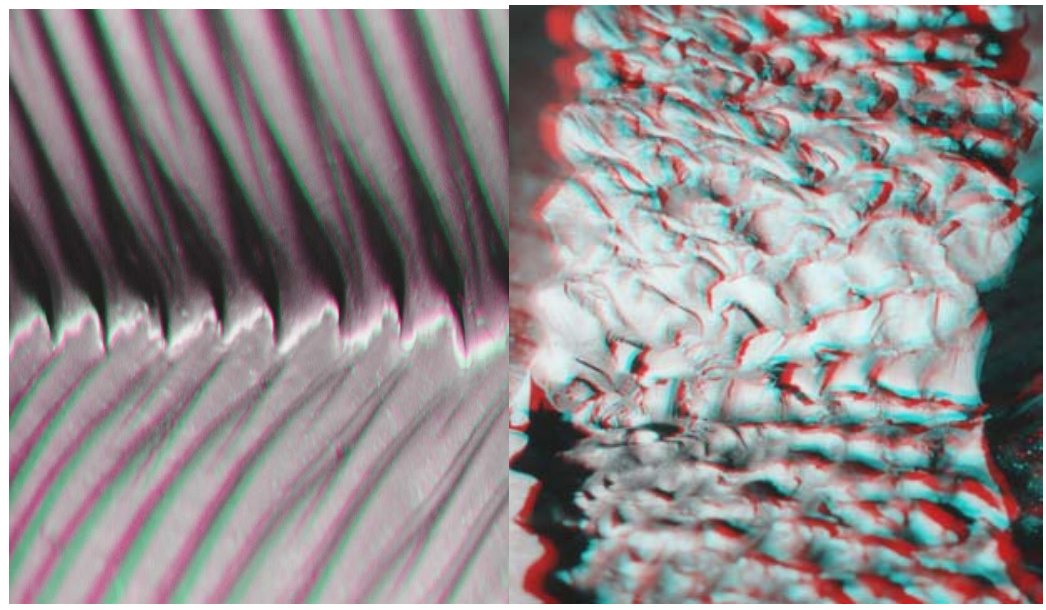

Fig 5: New Tire Ply Peel (Left) v. Old Tire Ply Peel (Right) All Images: 1mm = >---< 